\title{
Micrograms DDU Per Liter
}

National Cancer Institute

\section{Source}

National Cancer Institute. Micrograms DDU Per Liter. NCI Thesaurus. Code C161495.

A unit of equivalent concentration equal to the number of micrograms of $D$-dimer per unit volume equal to one liter. 